\title{
A Dual-Targeted Soybean Protein Is Involved in Bradyrhizobium japonicum Infection of Soybean Root Hair and Cortical Cells
}

\author{
Marc Libault, ${ }^{1}$ Manjula Govindarajulu, ${ }^{2}$ R. Howard Berg, ${ }^{2}$ Yee Tsuey Ong, ${ }^{1}$ Kari Puricelli, ${ }^{1}$ \\ Christopher G. Taylor, ${ }^{2}$ Dong Xu, ${ }^{3}$ and Gary Stacey ${ }^{1}$ \\ ${ }^{1}$ Division of Plant Sciences, National Center for Soybean Biotechnology, C.S. Bond Life Sciences Center, University \\ of Missouri, Columbia 65211 U.S.A.; ${ }^{2}$ Donald Danforth Plant Science Center, 975 North Warson Road, St. Louis 63132 \\ U.S.A.; ${ }^{3}$ Computer Science Department, C.S. Bond Life Sciences Center, University of Missouri, Columbia 65211, U.S.A.
}

Submitted 16 December 2010. Accepted 29 April 2011.

\begin{abstract}
The symbiotic interaction between legumes and soil bacteria (e.g., soybean [Glycine max L.] and Bradyrhizobium japonicum]) leads to the development of a new root organ, the nodule, where bacteria differentiate into bacteroids that fix atmospheric nitrogen for assimilation by the plant host. In exchange, the host plant provides a steady carbon supply to the bacteroids. This carbon can be stored within the bacteroids in the form of poly-3-hydroxybutyrate granules. The formation of this symbiosis requires communication between both partners to regulate the balance between nitrogen fixation and carbon utilization. In the present study, we describe the soybean gene $G m N M N a$ that is specifically expressed during the infection of soybean cells by $B$. japonicum. GmNMNa encodes a protein of unknown function. The $\mathrm{GmNMNa}$ protein was localized to the nucleolus and also to the mitochondria. Silencing of $G m N M N a$ expression resulted in reduced nodulation, a reduction in the number of bacteroids per infected cell in the nodule, and a clear reduction in the accumulation of poly-3-hydroxybutyrate in the bacteroids. Our results highlight the role of the soybean $G m N M N a$ gene in regulating symbiotic bacterial infection, potentially through the regulation of the accumulation of carbon reserves.
\end{abstract}

Legumes interact symbiotically with soil bacteria (rhizobia), leading to the development of a new root organ, the nodule. This symbiosis is initiated by the exchange of chemical signals between both partners, which determines the specificity of the host-symbiont interaction. More specifically, in response to flavonoids secreted by the plant, the bacteria synthesize a lipo-chitooligosaccharide known as a Nod factor (Lerouge et al. 1990). The perception of the Nod fac-

Current address for M. Govindarajulu: Genome Center and Department of Plant Sciences, University of California, Davis, 95616 U.S.A.

Current address for K. Puricelli: Midwest Research Institute, 425 Volker Blvd, Kansas City, MO 64110 U.S.A.

Current address for C.G. Taylor: Departments of Plant Pathology, Ohio State University, Wooster 44691 U.S.A.

Corresponding author: M. Libault; E-mail: libaultm@missouri.edu

* The $e$-Xtra logo stands for "electronic extra" and indicates that eight supplementary figures, five supplementary tables, and two videos are published online. tor by the plant leads to the deformation of root hair cells and to the activation of root cortical cell divisions leading to the formation of the nodule primordium. Inside the nodule, bacteria differentiate into bacteriods, which are able to fix atmospheric nitrogen for assimilation by the plant. In exchange, the plant provides a steady carbon supply to the bacteria, which can store this carbon in the form of poly-3-hydroxybutyrate (PHB) granules. Bacteroid PHB can subsequently be utilized as a carbon source or may also have other functions. For example, PHB controls the redox potential in the bacteroid; a consequence of the microaerobic conditions existing in the nodule that are necessary for optimal fixation of atmospheric nitrogen (Trainer and Charles 2006).

In past years, the functions of a limited number of legume genes involved in root hair cell infection by symbiotic bacteria have been defined based on forward and reverse genetic studies. These studies have relied heavily on the use of mutants derived from the two model legumes, Medicago truncatula and Lotus japonicus (Oldroyd and Downie 2008). However, as highlighted by Ferguson and Gresshoff (2009), soybean is now reemerging as a model in legume biology due to the release of the sequenced soybean genome (Schmutz et al. 2010), the development of routine methods for gene silencing (Govindarajulu et al. 2009; Libault et al. 2009, $2010 \mathrm{c}$ ), and the application of a variety of functional genomic methods (Libault et al. 2010a and b). By way of example, the soybean NFR1 and NFR5 genes, encoding the receptor kinases recognizing the Nod factor, were recently identified through the screening of a soybean mutant population (Indrasumunar et al. 2010, 2011). The analysis of the soybean transcriptome led to the identification of 233 genes consistently regulated at various times during root hair infection by Bradyrhizobium japonicum (Libault et al. 2010a). Among these genes, sequence comparisons failed to predict functions for 73 , due to the lack of sequence similarity to proteins with known functions. In this study, we investigated the role of one of these "proteins of unknown function", which we have named GmNMNa (nucleolar/mitochondrial protein involved in nodulation a). Our initial transcriptional analysis clearly established $G m N M N a$ as one of the most strongly induced root hair genes in response to B. japonicum inoculation (Libault et al. 2010a). Our study confirmed the strong induction of $G m N M N a$ expression during soybean root hair infection and localized expression using promoter reporter-gene fusions. RNA-mediated gene silencing of GmNMNa expression led to various phenotypes, including a decrease in nodule number, a 
reduction in the number of bacteroids per infected cells, and a decrease in the density of PHB granules in each bacteroid. Expression of a GmNMNa-green fluorescent protein (GFP) fusion in tobacco cells localized the protein to both the nucleolus and the mitochondria. Hence, one possibility is that GmNMNa plays an important role in mitochondrial function in the nodule, which may be manifested by effects on carbon metabolism reflected in fewer bacteroids and lower accumulation of carbon reserves (i.e., PHB). Indeed, earlier work showed that mitochondria accumulate at the periphery of legume infected cells to presumably maintain plant cell function under the low oxygen conditions of the nodule interior (Cermola et al. 2000; Day et al. 1986; Newcomb et al. 1985). Our data clearly suggest a role for $G m N M N a$ in regulating symbiotic bacterial invasion and survival during the early steps of soybean nodulation.

\section{RESULTS}

GmNMNa expression is induced in root hair cells in response to $B$. japonicum inoculation and during the early stages of soybean nodule development.

GmNMNa (Glyma01g45280) was initially identified as one of the most strongly upregulated genes during soybean root hair cell infection by B. japonicum (Libault et al. 2010a). These results suggest that $G m N M N a$ might play a critical role in the early infection of soybean root hair cells by B. japonicum.

Transcriptional induction of GmNMNa expression in response to B. japonicum inoculation was confirmed by quantitative reverse-transcription polymerase chain reaction (qRT-PCR) using mRNA isolated form inoculated and mock-inoculated (i.e., water-treated only) root hair cells isolated 0 to $72 \mathrm{~h}$ after inoculation (HAI) (Fig. 1A; Supplementary Table 1). GmNMNa

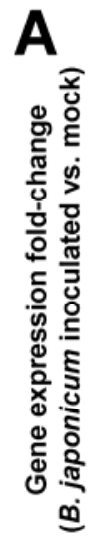

$10^{4}$

$10^{3}$

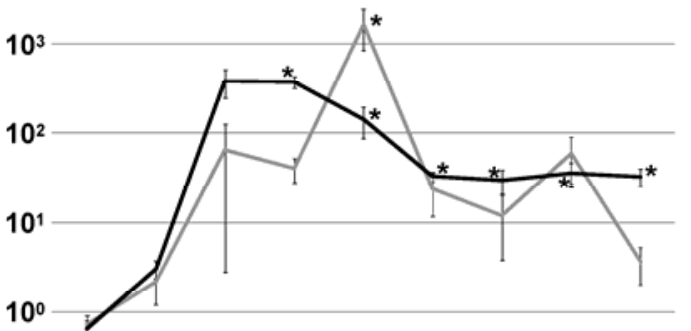

10

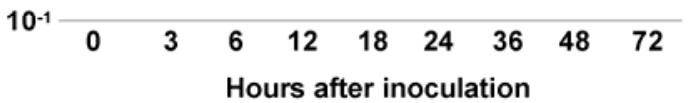

B

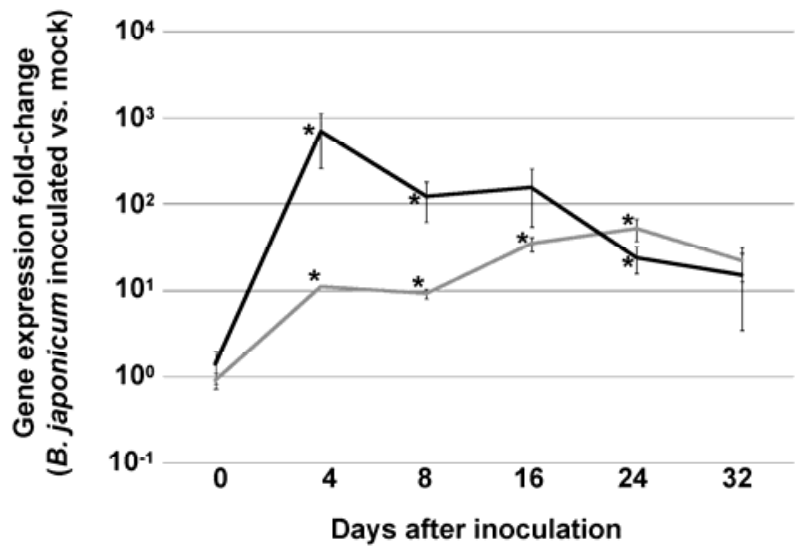

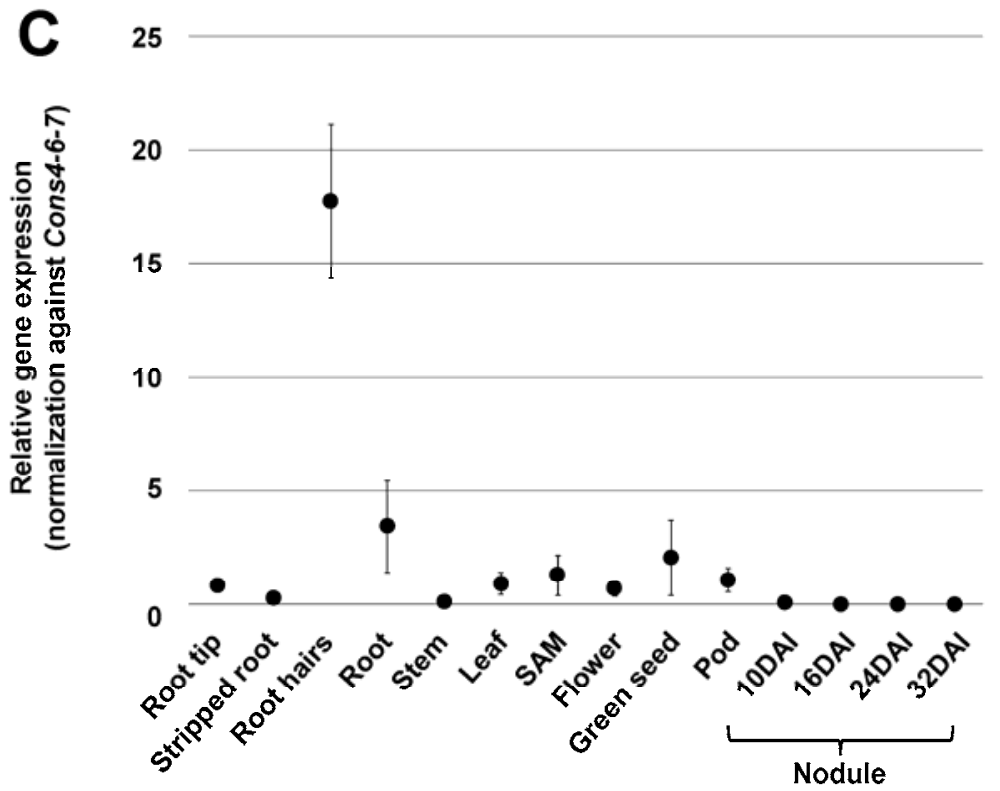

Fig. 1. Gene expression analysis of GmNMNa gene during the different steps of nodulation and in different soybean organs. The expression of the GmNMNa gene was quantified by quantitative reverse-transcription polymerase chain reaction (qRT-PCR) (black). ENOD4O (gray) was used as a positive control for the nodulation response. Previous to any analysis, the expression levels were normalized against the geometric mean of the soybean reference genes cons 4 , cons6, and cons7. A, Fold change in gene expression during root hair infection between Bradyrhizobium japonicum-inoculated root hairs versus mockinoculated root hairs. B, Fold change in gene expression during nodule development between $B$. japonicum-inoculated roots versus mock-inoculated roots. C, Gene expression levels were quantified in 14 different soybean tissues, including isolated nodules (10, 16, 24, and 32 days after inoculation [DAI]). At 24 DAI, GmNMNa expression was not detected by qRT-PCR. The different time points and tissues tested are shown on the $x$ axis, while the $y$ axis presents the fold changes (A and B) or the normalized expression levels (C) for each gene analyzed. Average relative expression levels of each gene across three replicates in each tissue are shown on the $y$ axis. Standard error bars are shown. Asterisk indicates Student's $t$ test $<0.05$ when comparing inoculated/nodulated versus mock-inoculated/nodule-free tissues. 
expression was also measured in nodulated and mock-inoculated roots (0 to 32 days after inoculation [DAI]) (Fig. 1B). In both analyses, GmENOD40 was used as a positive control for the infection response (Minami et al. 1996) and gene expression levels were normalized against the geometric mean of the soybean reference genes cons 4 , cons6, and cons7 (Libault et al. 2008). Consistent with our previous results (Libault et al. 2010a), GmNMNa expression was strongly induced during root hair infection, with the highest expression occurring between 6 and 18 HAI (>100-fold changes), and during nodule development, with the highest expression occurring at 4 DAI (approximately 700-fold change). A strong decrease in GmNMNa expression occurred at later time points (24- and 15-fold changes at 24 and 32 DAI, respectively). Compared with the GmENOD40 expression profile where maximum induction occurred during later nodule development, the maximum expression of $G m N M N a$ was restricted to the earliest stages of soybean nodulation (Fig. 1A and B).

To investigate the possible expression of GmNMNa in other parts of the plant, we consulted the two independent soybean transcriptome atlases (Libault et al. 2010b; Severin et al. 2010), which present gene expression results from 28 different tissues and conditions as measured by high-throughput cDNA sequencing. This analysis showed that GmNMNa expression was exclusively restricted to soybean root hair cells independent of $B$. japonicum inoculation (Supplementary Table 2). The absence of GmNMNa expression in mature nodules, which were used during generation of the soybean expression atlases, is consistent with the drop of expression observed during nodule development (Fig. 1B). To confirm the GmNMNa expression pattern across different tissues and to better understand the regulation of its expression in soybean nodules, we performed qRT-PCR in 14 different soybean tissues, including nodules isolated at different days after B. japonicum inoculation (i.e., root tip, stripped root [root devoid of root hairs], root hair, root, stem, leaves, shoot apical meristem, flower, green seed, young pods, and 10-, 16-, 24-, and 32-DAI nodules]. With the exception of the root hair cells and the mature root, GmNMNa was expressed at very low levels in all soybean tissues; including nodules (Fig. 1C). Interestingly, GmNMNa was expressed at a higher level in 10-DAI nodules compared with mature nodules (16-, 24-, and 32-DAI nodules) (Fig. 1C). We assumed that the detection of GmNMNa transcripts in the mature root reflects its overall expression in root hair cells rather than the entire root system, based on the fact GmNMNa transcripts were barely detected in stripped roots (i.e., roots devoid of root hair cells). Similarly, induction of $G m N M N a$ expression in nodulated roots (Fig. 1B) likely reflects the strong expression of GmNMNa in infected root hair cells (Fig. 1A) and emerging nodules. Altogether, our data suggest that GmNMNa expression is restricted to the root hair cell and is specifically induced during root hair cell infection by $B$. japonicum and, at a lower level, during the earliest stages of soybean nodule development.

To further localize the site of GmNMNa expression, transgenic soybean roots expressing GFP and $\beta$-glucuronidase (GUS) under the control of the GmNMNa promoter (i.e., approximately 2,000 bp located upstream of the GmNMNa ATG codon) were monitored during B. japonicum inoculation. GFP signal and GUS staining were not observed in mock-inoculated transgenic roots. However, the GFP signal was clearly seen in a subset of the root hair cells in response to $\mathrm{B}$. japonicum inoculation, confirming the induction of GmNMNa in these cells (Supplementary Fig. 1). Interestingly, these GFPpositive root hair cells appeared to be deformed or curled, perhaps indicative of the response to B. japonicum infection (Fig. 2A). During nodule development, the GmNMNa expression pattern changed considerably. In immature nodules, GmNMNa expression was restricted to the center of the emerging nodule, which is the location of the cells newly infected by $B$. japonicum (Fig. 2B). Later, in mature nodules, GmNMNa expression was observed in the uninfected cortical cells but at a very low level (Fig. 2C). Altogether, GmNMNa expression was stronger in developing nodules than in mature nodules (Fig. 2B and C), confirming the qRT-PCR results described above.

\section{The NMN family is conserved in legumes.}

The recent release of the soybean genome sequence (Schmutz et al. 2010) and the nearly complete barrel medic and lotus genome sequences are useful tools to better understand legume biology and evolution. These sequences were mined to identify potential $G m N M N a$ orthologs, as well as possible paralogs in the soybean genome. For further comparison, we also sought GmNMNa homologous genes in the Arabidopsis thaliana genome.

Comparison of the GmNMNa protein sequence against the soybean genome identified one putative homolog on chromosome 11 (from 118,735 to 119,219 bp; tblastn, score: 276, $e$ value: $4.8 \mathrm{e}^{-72}$ ). Based on the published genome annotation, no gene was predicted at this location. However, this is not surprising because several expressed but currently unannotated genes were identified through high-throughput sequencing of soybean cDNA (Libault et al. 2010a and b). Indeed, examination of the soybean transcriptome atlas (Libault et al. 2010b) identified a transcript corresponding to the GmNMNa homolog (i.e., $G m N M N b$ ) located on the chromosome 11 (from 118,691 to $119,156 \mathrm{bp}$ ), confirming the transcriptional activity of this gene. Similarly to GmNMNa, GmNMNb was also expressed in inoculated root hair cells but at a significantly lower level compared with GmNMNa. Using FGENESH (Salamov and Solovyev 2000), a protein composed of 234 amino acids lacking known protein domains was predicted to be encoded by $G m N M N b$. GmNMNa and GmNMNb appear to lie within regions of clear homology based on a comparison of syntenic regions found on soybean chromosome 1 and 11 (Libault et al. 2010b) (Supplementary Table 3; Supplementary Fig. 2).

The GmNMNa protein sequence was also compared against the L. japonicus, M. truncatula, and A. thaliana coding sequences. In $A$. thaliana, no GmNMNa homolog was identified (i.e. blastp; best hit: At05g65925, score $=33, e$ value $=0.067$ ). In L. japonicus, a putative ortholog encoding a protein of 132 amino acids was identified (LjCM0200-133; tblastn, score $=60$, $e$ value $\left.=4 \mathrm{e}^{-09}\right)$. This gene was not represented on the array used to establish the transcriptome atlas of L. japonicus (Høgslund et al. 2009). One $M$. truncatula predicted gene (i.e., Med$\operatorname{Tr} 05 \mathrm{~g} 005450)$, encoding a protein of 172 amino acids, shared significant similarity to GmNMNa (blastp, $e$ value $=3 \mathrm{e}^{-19}$ ). Based on synteny analyses, this gene is a likely orthologous to $G m N M N a$. Indeed, consistent with this hypothesis, expression of MedTr05g005450 (i.e., MtNMN, Affymetrix probe set Mtr.26415.1.S1_at), as shown in the M. truncatula gene expression atlases (Benedito et al. 2008; He et al. 2009), was elevated during nodulation and in mycorrhizal roots, suggesting a role for this gene in plant-microbe symbiosis (Supplementary Fig. 3).

The GmNMNa protein sequence was also compared against the National Center for Biotechnology Information nonredundant protein sequence (blastp) and DNA sequence (tblastn) databases. Several putative homologs were identified but only in plant species, suggesting that the $N M N$ family may be a plant-specific gene family (Supplementary Table 4). However, the overall poor scores and $e$ values that resulted from this analysis suggest that the NMN-like protein sequences have rapidly diverged during plant evolution.

Given the lack of functional annotation for GmNMNa, we used MaliP software to identify conserved domains. The N-ter- 
minal amino acid sequence located on the N-terminal end of NMN proteins was poorly conserved. However, the 40 amino acids located at the NMN C-terminal end were strongly conserved and enriched in charged amino acids (e.g., aspartate, glutamate, lysine, and arginine) and tyrosine (Supplementary Fig. 4). Altogether, these amino acids represented $55 \%$ of the 40 conserved amino acids on the $\mathrm{C}$ terminal of NMN proteins. The function of this conserved domain is unknown.

\section{Multiple subcellular localization of GmNMNa.}

$G m N M N a$ is predicted to encode a protein of 147 amino acids. We assume that the GmNMNa protein sequence is the full-length protein for two reasons: i) the GmNMNa transcript was predicted with high confidence based on the identification of its full-length cDNA sequence (Schmutz et al. 2010) and ii) the similarities between the size and the amino acid sequences between GmNMNa, MtNMN, and LjNMN proteins strongly support the idea that these proteins are full-length proteins.

Analysis of the GmNMNa amino acid sequence revealed the presence of a putative mitochondrial targeting signal (MTS) at the $\mathrm{N}$ terminus, as identified using iPSORT $\left({ }^{9} \mathrm{KQSKLKH}^{15}\right.$ )
(Bannai et al. 2002) and PSORT software $\left({ }^{21} \mathrm{MRILKK}^{26}\right)$. Interestingly, the ${ }^{9} \mathrm{KQSKLKH}{ }^{15}$ MTS is strongly conserved between GmNMNa, GmNMNb, MedTr05g005450, and LjCM0200-133 proteins.

To confirm these bioinformatic predictions, we expressed the fusions between the GmNMNa and the GFP cDNAs under the control of the dual $35 \mathrm{~S}$ promoter in tobacco leaf cells by infiltrating Agrobacterium tumefaciens strains carrying the plasmids of interest. As a control, GFP alone was expressed in tobacco leaves.

The GFP alone was localized in the cytoplasm and in the nucleus of tobacco cells, with the exception of the nucleolus (Fig. 3A) (Libault et al. 2010c). When fused to GmNMNa, the GFP signal was specifically detected in the nucleolus (Fig. 3B through D; Supplementary Videos 1 and 2). We hypothesized that the nuclear localization of the GFPGmNMNa protein is probably due to its passive diffusion through the nuclear membrane (i.e., the predicted $47-\mathrm{kDa}$ size of GFP-GmNMNa chimeric protein is below the exclusion limit for plant nuclei [i.e., 40 to $60 \mathrm{kDa}$ ) (Berg and Beachy 2008). However, the nucleolar localization of
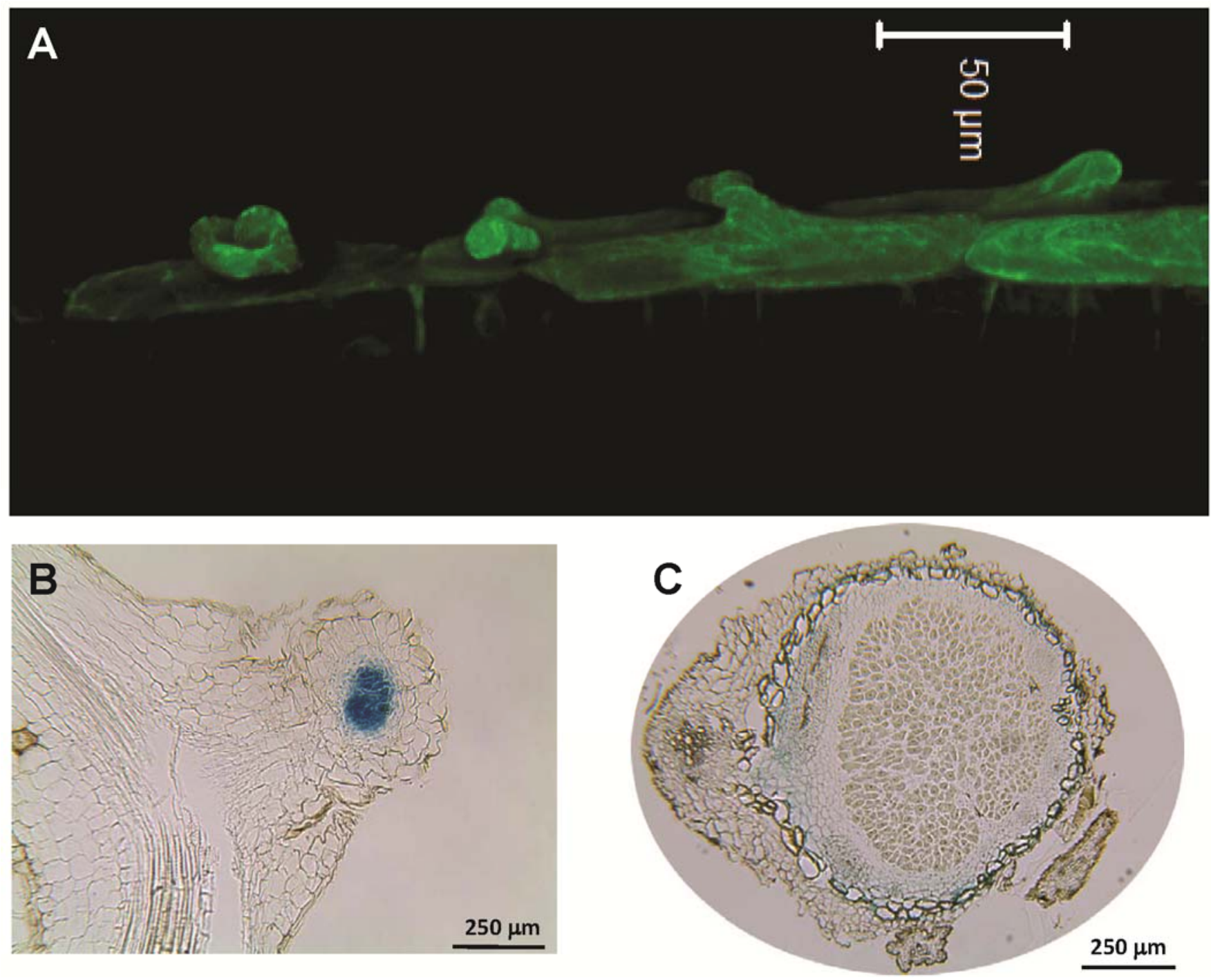

Fig. 2. Expression pattern of GmNMNa during root hair infection by Bradyrhizobium japonicum and soybean nodule development. GmNMNa promoter sequence was fused upstream to the green fluorescent protein (GFP) and $\beta$-glucuronidase (GUS) reporter genes. A, Representative expression patterns of $G m N M N a$ promoter-GFP in the soybean whole root 7 days after $B$. japonicum inoculation. At this time, soybean transgenic root hair cells are infected by $B$. japonicum. B and C, Representative expression patterns of GmNMNa promoter-GUS in nodule cross sections 28 days following B. japonicum inoculation. At this stage, transgenic roots showed a mixture of immature (B) and mature nodules (C). Confocal (A) and light microscope (B and C) images are shown. Bars $=50 \mu \mathrm{m}(\mathrm{A})$ and $250 \mu \mathrm{m}(\mathrm{B}$ and $\mathrm{C})$. 
GmNMNa could reflect either its true biochemical role, because GFP alone was excluded from the nucleolus (Fig. 3A), or the nonspecific binding of GmNMNa protein to the charged (basic) nucleolar compartment.

In addition to its localization in the nucleolus, GFP fluorescence was also seen in discrete cytosolic dots when GFP was fused to the $\mathrm{C}$ terminus of GmNMNa (Fig. 3B). In comparison, this localization was not seen when the GFP was fused to the $\mathrm{N}$ terminus of GmNMNa, presumably due to blocking of the putative mitochondrial targeting signal (Fig. 3C). These spots appeared to co-localize with mitochondria. To further investigate this localization, we co-expressed the GmNMNa-GFP fusion with the mt-rk plasmid (Nelson et al. 2007) carrying a translational fusion between an MTS and mCherry in tobacco leaves. We verified the absence of cross contamination between the GFP and mCherry fluorescent signals with the set of filters used on the confocal microscope (Supplementary Fig. 5). A clear overlap of the GFP and mCherry signals was observed, supporting the idea that the GmNMNa protein was targeted to the mitochondria (Fig. 3E).

\section{Silencing of GmNMNa reduces nodule number, bacteroid density, and the accumulation of polyhydroxybutyrate.}

To characterize the role of GmNMNa during nodulation, we generated soybean transgenic roots expressing a $G m N M N a$ RNA interference (RNAi) construct using the hairy root transformation method (Govindarajulu et al. 2009; Libault et al. 2009, 2010c). The RNAi construct consisted of a DNA fragment derived from the $3^{\prime}$ untranslated region (UTR) of GmNMNa designed to form a double-stranded RNA. The 3' UTR region of GmNMNa was selected in order to maximize the specificity of the RNAi construct. Comparison of this sequence against the soybean genome confirmed its specificity against $G m N M N a$ (score $=299.8, e$ value $=7.3 \mathrm{e}^{-80}$; secondbest hit: $G m N M N b$, score $=69.9, e$ value $\left.=1.2 \mathrm{e}^{-10}\right)$. Based on the overall very low expression of $G m N M N b$, we assume that our RNAi construct will predominantly affect GmNMNa mRNA abundance.

The number of nodules on 45 RNAi-GmNMNa and 75 GUS-RNAi control transgenic roots was counted 42 DAI using three independent biological replicates. Past experience indi-

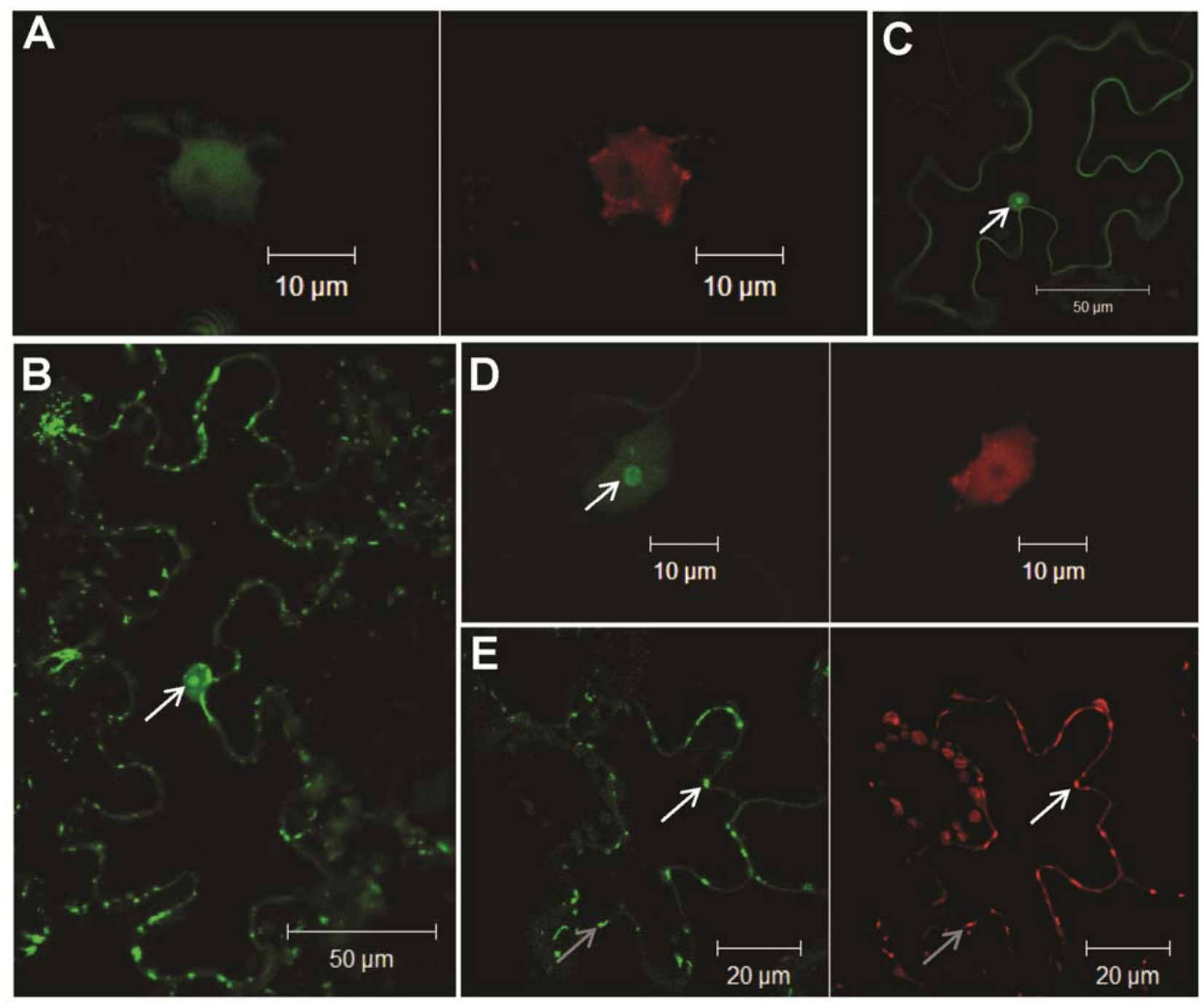

Fig. 3. In vivo observation of the mitochondrial and nucleolar localization of GmNMNa. Epidermal cells of tobacco leaves expressing GmNMNa protein fused to the green fluorescent protein (GFP) were observed with a confocal microscope. A, As a control, tobacco leaves expressing the GFP alone (green) were also stained with propidium iodide (red). Independently of the direction of the fusions between GmNMNa and the GFP (B, GmNMNa-GFP; $\mathbf{C}$ and $\mathbf{D}$, GFP-GmNMNa), GmNMNa was targeted to the nucleolus (white arrows). To confirm this nucleolar localization, the nucleolus was stained with propidium iodide (C, red). Depending on the orientation of the translational fusion, GmNMNa was targeted (B, GmNMNa-GFP) or not (C, GFP-GmNMNa) to plant cell organelles. E, Localization of GmNMNa to the mitochondria was confirmed (white and gray arrows) by co-transforming epidermal tobacco cells with the GmNMNa-GFP (green) and a mitochondrial targeting signal-mCherry fusions (red). 
cates that transgenic hairy roots nodulate more slowly than wild-type roots and, as a consequence, 42-DAI soybean hairy roots contain a mixture of developing and mature nodules. On average, the RNAi-GmNMNa transgenic roots produced approximately $60 \%$ fewer nodules than the control GUS-RNAi transgenic roots. Silencing of GmNMNa appeared to block nodule development and not initiation because the total number of nodule primordia was not significantly different between the silenced and control transgenic roots (Fig. 4A; Student's $t$ test, $P$ value $=0.47)$. However, there was a significant reduction in the number of developing and mature nodules formed on the RNAi$G m N M N a$ transgenic roots (Fig. 4A; developing nodules, $P$ value $<0.002$; mature nodules, $P$ value $<0.02)$. Microscopic analysis of nodule sections showed that there was no change in the cellular organization of the nodule primordia or mature nodules as a result of GmNMNa silencing. In addition, GmNMNa-
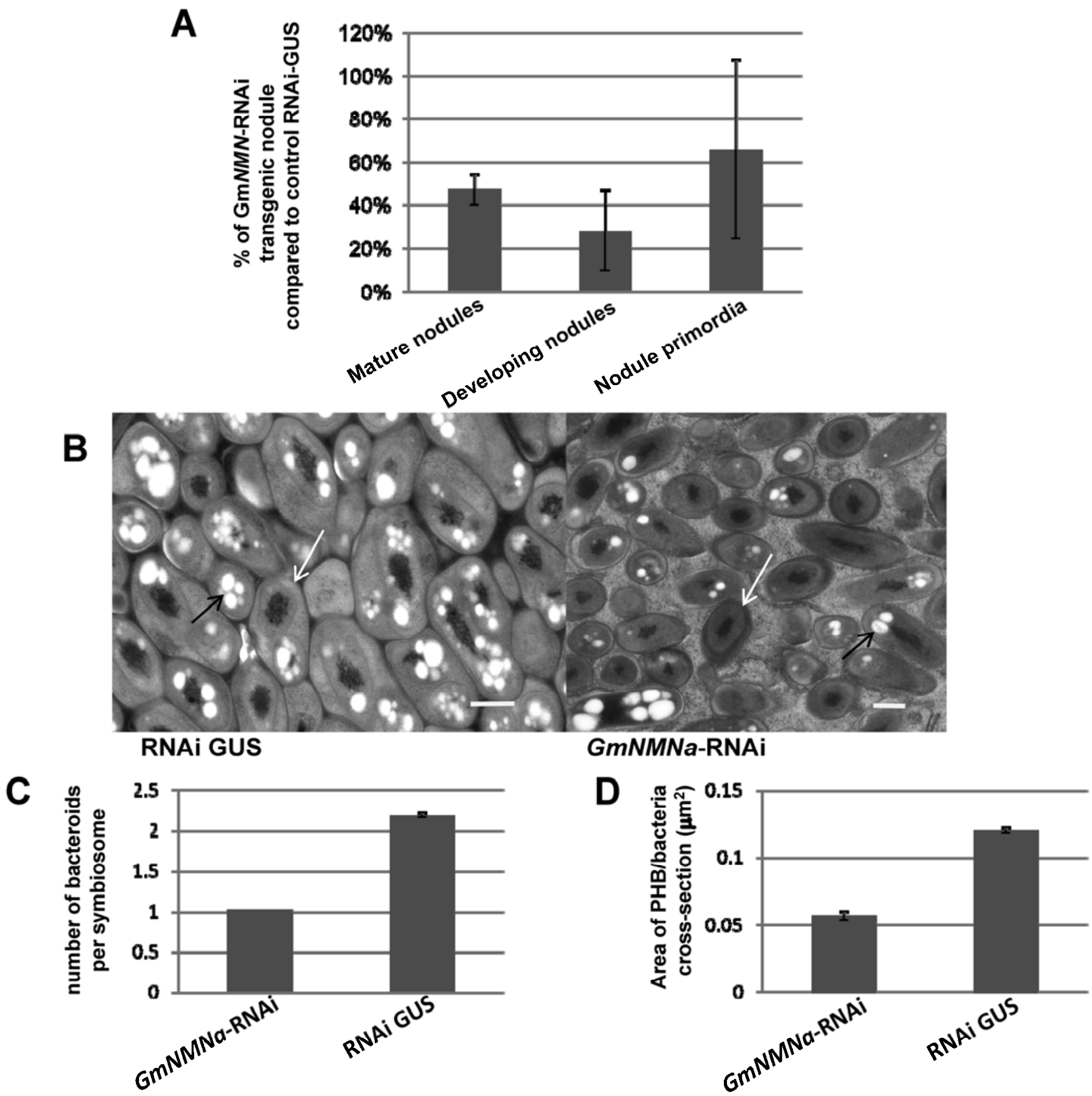

Fig. 4. Silencing of GmNMNa reduces poly-3-hydroxybutyrate (PHB) granule accumulation, bacteroid per infected cell, and nodule number. A, Number of nodules formed on GmNMNa-RNAi (RNA interference) transgenic roots as a percentage of nodules formed on control RNAi- $\beta$-glucuronidase (GUS) roots. Nodules were categorized by their stage of development. Green fluorescent protein (GFP) was used as a reporter gene to identify the transgenic roots. Nodule numbers were counted on 45 and 75 GmNMNa-RNAi and GUS-RNAi transgenic roots, respectively. Standard error bars are shown. B, Representative transmission electron micrographs of infected cells of soybean mature GUS-RNAi (left picture) and GmNMNa-RNAi (right picture) nodules. Bacteroids (white arrows) were observed in both GUS-RNAi and GmNMNa-RNAi nodules but were found in lower abundance in GmNMNa-RNAi transgenic nodules. In addition, a significant decrease in PHB accumulation was observed in GmNMNa-RNAi bacteroids (black arrows). Bars $=0.5 \mu \mathrm{m}$. C, Average number of bacteroids per symbiosome in infected cells of GmNMNa-RNAi and GUS-RNAi transgenic nodules. A Student's $t$ test $=0$ was calculated when comparing both sets of infected cells. Standard error bars are shown. D, Average area of PHB particles per bacteroid in GmNMNa-RNAi- and GUS-RNAi-infected cells of transgenic nodules. A Student's $t$ test $\left(P\right.$ value $\left.<1 . \mathrm{e}^{-36}\right)$ was calculated when comparing both sets of bacteroids. Standard error bars are shown. 
RNAi mature nodules exhibited a normal level of leghemoglobin expression, suggesting that they were fully functional.

Electron microscopy showed that both the RNAi-GmNMNa and GUS-RNAi root nodules were infected by B. japonicum (Fig. 4B). Infection threads could be easily seen in RNAiGmNMNa nodules; again, suggesting no significant effect on the infection process (Supplementary Fig. 6A). However, the number of bacteroids per symbiosome in infected cells was strongly reduced in RNAi-GmNMNa nodules (Fig. 4B and C; bacteroid number from 1,915 and 1,302 RNAi-GmNMNa and GUS-RNAi infected cells, respectively, were counted; Student's $t$ test $=0$ ). These results were confirmed through observation of sections of RNAi-GmNMNa and GUS-RNAi nodules stained with SYTO13 fluorescent dye. As previously described by Veereshlingam and associates (2004), SYTO13 dye preferentially stains bacteroids, making this dye ideal to observe a potential defect in nodule infection as well as infection thread formation. In addition to the reduction in the number of bacteroids per symbiosome in infected cells, the number of PHB granules per bacteroid was also significantly reduced in bacteroids seen in the RNAi-GmNMNa nodules (Fig. 4B and D; PHB area [in square micrometers] from 201 and 635 RNAiGmNMNa and GUS-RNAi bacteroids, respectively, was measured; Student's $t$ test $\left.<1 . \mathrm{e}^{-36}\right]$.

To confirm the decrease of GmNMNa transcripts in silenced roots, we quantified GmNMNa expression by using qRT-PCR in RNAi-GmNMNa and GUS-RNAi control roots. As expected based on our previous qRT-PCR experiments, GmNMNa expression was detected at a very low level in the 42-DAI transgenic roots. However, we clearly quantified a lower expression of $G m N M N a$ in the RNAi-GmNMNa transgenic roots compared with the control roots: GmNMNa expression was detected in a higher number of RNAi-GUS control roots compared with the RNAi-GmNMNa roots. When detected, GmNMNa transcripts were 2.4 times less abundant in RNAi-GmNMNa roots compared with the RNAi-GUS control roots (Supplementary Fig. 7). To address the question of the specificity of the RNAi construct, we quantified the expression of $G m N M N b$ in the RNAi-GmNMNa and GUS-RNAi transgenic roots by performing qRT-PCR. Independent of the roots analyzed, we were unable to detect expression of the GmNMNb transcript. To better characterize the expression profile of $G m N M N b$, we also quantified $G m N M N b$ expression across various soybean tissues, including inoculated and mock-inoculated root hair cells. The lack of expression of $G m N M N b$ across soybean tissues strongly supports the soybean transcriptome atlas dataset, showing that $G m N M N b$ expression was consistently very low. Altogether, the lack of expression of $G m N M N b$ indicates that the nodule phenotype of RNAi-GmNMNa roots was most likely due to the specific silencing of GmNMNa expression.

\section{DISCUSSION}

As is the case for all sequenced genomes, a significant percentage $(17 \%)$ of the predicted 46,430 protein-encoding genes in the soybean genome lacks functional annotation or even identified protein domains of unknown function. Previous transcriptome analysis identified 73 genes of unknown function, from a total of 233 soybean root hair genes that specifically responded to B. japonicum inoculation (Libault et al. 2010a). Defining a mechanistic role for these genes in the nodulation process is difficult because there is no obvious starting point for such an analysis.

In the case of GmNMNa, we used a variety of molecular approaches to define the cellular location of the protein and its expression pattern and phenotype after RNAi silencing to deduce its possible role in soybean nodulation.

\section{Silencing of $G m N M N a$ reduces nodulation and impacts bacteroid development.}

In plant-bacteria symbiosis, differentiated bacteria fix and assimilate atmospheric nitrogen for the plant. In return, the plant provides a carbon source to the bacteroid. The sucrose molecules produced by plant photosynthesis are transported to the nodule and degraded through the glycolysis pathway and the TCA cycle leading to the synthesis of dicarboxylic acids (e.g., malate), which are thought to serve as the primary carbon source for the bacteroid (Benedito et al. 2010; Ronson et al. 1981; Udvardi and Day 1997). Excess carbon is stored within the bacteroids in the form of PHB particles. In soybean, PHB is not used as a source of energy to maintain nitrogenase activity (Wong and Evans 1971). Previously, Lodwig and associates (2005) hypothesized that PHB accumulated in the rhizobial symbiont to be used during infection of the plant root hair cells and differentiation into bacteroids. This last hypothesis is consistent with the reduction in nodule and bacteroid development seen upon silencing of $G m N M N a$ expression. Indeed, the localization and timing of GmNMNa expression is consistent with a role during the early events of nodulation. The impact of GmNMNa silencing on PHB accumulation may be the result of defective carbon metabolism (perhaps through disruption of mitochondrial function) or some other cause. However, the reduction of PHB accumulation in the bacteroids of the GmNMNa silencing nodules may be sufficient to explain the nodulation phenotype. For example, Wang and associates (2007) reported a significant delay in nodulation and nitrogen fixation when alfalfa roots were inoculated with a Sinorhizobium meliloti phbC mutant defective in PHB biosynthesis.

GmNMNa is targeted to two distinct cellular compartments.

Only a few examples of multiple targeted plant proteins have been confirmed in vivo (Carrie et al. 2009; Mitschke et al. 2009; Schwacke et al. 2007). Carrie and associates (2009) reported a total of 49 dual-targeted Arabidopsis thaliana proteins. Among them, 42 of the 49 were localized in the mitochondria and in the chloroplast due to the redundancy of biochemical pathways between the two organelles. Bioinformatic analysis based on possible targeting signals suggests that a significant number of dual-targeted proteins might be present in plants. For example, analysis of the predicted Arabidopsis proteome suggested that 523 Arabidopsis proteins are targeted to more than one subcellular compartment (Mitschke et al. 2009). Localization of GmNMNa-GFP translational fusions supports the targeting of this protein to both the nucleolus and mitochondria. The latter is supported by the prediction of two putative MTS at the $\mathrm{N}$ terminus of GmNMNa. The interpretation of the nucleolar localization of GmNMNa remains unclear: it could reflect the true biochemical role of GmNMNa because GFP alone was excluded from the nucleolus (Fig. 3A) or it could reflect nonspecific binding to the charged nucleolar compartments. This latter interpretation would suggest that the major site of GmNMNa function is in the mitochondria.

Dual targeting to both the mitochondria and nucleus is not well characterized in plants. For example, only one Arabidopsis gene (At1g79430), encoding the ALTERED PHLOEM DEVELOPMENT (APL)-like MYB transcription factor, was reported to be targeted to the mitochondria (Carrie et al. 2009) and to the nucleus (Bonke et al. 2003). This differential localization was dependent on which transcript variant of APL was fused to the GFP. When the GFP was fused to the $\mathrm{N}$ terminus of the longest APL transcript (358 amino acids), the translational protein was exclusively localized in the nucleus (Bonke et al. 2003). In contrast, when GFP was fused to the C terminus of the shortest APL transcript variant (293 amino acids), 
the chimeric protein was localized exclusively to the mitochondria (Carrie et al. 2009). According to Carrie and associates (2009), the differential subcellular localization of APL proteins is dependent of the change of the relative localization of an MTS at the N terminus of APL. In a previous study, de Castro Silva Filho and associates (1996) also noticed that the relative $\mathrm{N}$-terminal location of MTS was important to properly target a protein to the mitochondria.

An examination of the non-plant literature identified the eukaryotic ribonucleoporin endoribonuclease RNase MRP protein as another example of a protein localized to the nucleolus and mitochondria. In the nucleus, this protein is involved in the cleavage of the ribosomal RNA (rRNA) precursor (Lindahl and Zengel 1995; Tollervey 1995). In the mitochondria, RNase MRP is involved in the formation of the primers necessary for the replication of the mitochondrial DNA (Chang and Clayton 1987; Gold et al. 1989).

Given the similar subcellular localization of GmNMNa and RNase MRP, we explored the possibility that GmNMNa might bind to RNA. Indeed, analysis of the GmNMNa protein sequence predicted three putative nucleic-acid-binding domains using three independent bioinformatic tools: DNABindR (Yan et al. 2006), BindN (Wang and Brown 2006), and DP-Bind (Hwang et al. 2007) (Supplementary Fig. 8). In addition, using another bioinformatic tool, SVM-Prot (Cai et al. 2003), GmNMNa was predicted to interact with rRNA. In order to test this directly, we purified the hemagglutinin-tagged GmNMNa protein from transgenic tobacco leaves and tested the ability of this protein to bind to rRNA (data not shown). However, these experiments failed to reveal any significant RNA binding and, therefore, the role of GmNMNa as an RNAbinding proteins remains hypothetical.

\section{Conclusion.}

It is common to identify a significant number of proteins of unknown function when conducting transcriptomic studies. These proteins are particularly difficult to characterize functionally due to the lack of any predicted starting point for such an analysis (e.g., a specific predicted enzymatic activity). GmNMNa is among the most strongly upregulated genes during the early events of root hair infection by $B$. japonicum and, therefore, was predicted to play an important role in symbiotic development. The nodulation phenotypes seen upon GmNMNa silencing supported this notion and also revealed a strong effect on the abundance of bacteroids in the nodule and their physiology (i.e., as revealed by a marked reduction in PHB content). Localization of a GmNMNa-GFP fusion indicates that this protein is targeted to both the nucleolus and mitochondria. The latter might suggest a direct role in nodule respiration, which could impact bacteroid carbon metabolism.

\section{MATERIALS AND METHODS}

\section{Identification of GmNMNa homologous and orthologous genes.}

To identify GmNMNa homolog and orthologs, the GmNMNa protein sequence was blasted against the Glycine max (tblastn), M. truncatula (blastp), L. japonicus (tblastn), and A. thaliana (blastp; The Arabidopsis Information Resource) genomes. Synteny analyses between GmNMNa, GmNMNb, and their M. truncatula orthologs was highlighted based on the order, content, and direction of genes located on more than $100,000 \mathrm{bp}$ of genomic sequences. A $P$ value $<\mathrm{e}^{-20}$ was used as a cut-off in the Blast analyses between gene sequences. $\mathrm{Mi}$ crosynteny relationships were generated by using the CMTV software (Sawkins et al. 2004).

\section{Bacterial cultures.}

Escherichia coli, Agrobacterium rhizogenes, and B. japonicum USDA110 were grown and washed as described by Libault and associates (2009). A. tumefaciens (strain C58C1 [pCH32]) was grown as described by Libault and associates (2010c).

\section{Cloning.}

GmNMNa promoter cloning (i.e., $2 \mathrm{~kb}$ of nucleotide sequences located upstream of the first codon encoding $\mathrm{GmNMNa}$ protein) upstream of the GFP and GUS cDNAs was performed as described by Libault and associates (2010c). Primers used to amplify the GmNMNa promoter with the Gateway compatible AttB boxes using soybean 'Williams 82' genomic DNA as template were GmNMNapromoAttB-for (AAAAAGCAGGCT GACAAGTGAATTTAATTTGATTTTTTCT) and GmNMNapromoAttB-rev (AGAAAGCTGGGTACTGAAATGGTCGAT GATGAAAG) first and the universal AttB primers (attB1adaptor [GGGGACAAGTTTGTACAAAAAAGCAGGCT] and attB2adaptor [GGGGACCACTTTGTACAAGAAAGCTGG GT]) second.

Using Gateway BP and LR Clonase II enzyme mixes (Invitrogen, Carlsbad, CA, U.S.A.), the GmNMNa promoter fragment was introduced into the pDONR-Zeo vector (Invitrogen), then into pYXT1 or pYXT2 destination vectors carrying the GUS and GFP reporter genes, respectively (Xiao et al. 2005).

Similarly, two-step PCR (i.e., GmNMNacDNAAttB-for [AAAAAGCAGGCTATATGAGAAAGGAATTAGCAAAGAA GAA] and GmNMNacDNAAttB-rev [AGAAAGCTGGGTTA ACAGGCACAGGGCGGCTGT] primers, and then, attB1adaptor and attB2adaptor primers were used to amplify $G m N M N a$ cDNA with the AttB boxes) followed by the use of the Gateway system led to the creation of the translational fusion between GmNMNa and GFP cDNAs into pMDC43 and pMDC83 vectors (Curtis and Grossniklaus 2003).

To silence GmNMNa, we PCR-amplified a 244-bp fragment specific to the 3' UTR region of GmNMNa cDNA by using the primers $G m N M N$-RNAifor (GAACGAAAGTCAATCAAGAT ATCAAA) and $G m N M N$-RNAirev (GAGCGCCATAGGAATG TCAC). This PCR product was cloned into the entry vector CGT 11050, then into the destination vector CGT 11017A (Libault et al. 2009) as described by Libault and associates (2010c). The resulting $G m N M N a$-RNAi vector and the RNAi control vector CGT 5200 (Govindarajulu et al. 2009) were electroporated into A. rhizogenes K599 and used for composite plant production.

pYXT1-GmNMNa promoter, pYXT2-GmNMNa promoter, CGT11017-GmNMNa-RNAi, and CGT5200 vectors were used to transform A. rhizogenes (strain K599) while A. tumefaciens [strain C58C1 (pCH32)] was transformed with GmNMN$a \mathrm{cDNA}-\mathrm{pMDC} 43$, GmNMNacDNA-pMDC83, and pMDC43 vectors.

\section{Plant culture and treatment.}

Isolated root hair cells inoculated with $B$. japonicum and mock-inoculated, nodulated, and nodule-free roots and the different soybean tissues were produced as described by Libault and associates (2009, 2010a and b). At least three independent biological replicates per condition were harvested to ensure reproducibility of the plant tissues analyzed.

\section{Plant transformation and microscopy.}

A. tumefaciens strains carrying GmNMNacDNA-pMDC43, GmNMNacDNA-pMDC83, and the negative control pMDC43 vectors were used to transform Nicotiana benthamiana leaves. The GFP localization was observed with a Zeiss LSM 510 META confocal microscope 3 days after leaf infiltration (488$\mathrm{nm}$ excitation wavelength and 500- to 550-nm emission filter). 
Co-localization experiments between GmNMNa and mitochondria were performed by co-transforming tobacco leaves with the GmNMNa-GFP constructs described above and CD3992 vector carrying a translational fusion between a mitochondria targeting signal and the mCherry red fluorescent protein (Nelson et al. 2007). In addition, when needed, nuclei were stained by using propidium iodide $(5-\mu \mathrm{g} / \mathrm{ml}$ final concentration, 543-nm excitation wavelength, and 565- to $615-\mathrm{nm}$ emission filter).

Soybean hairy root transformation was performed as described by Libault and associates (2009) using A. rhizogenes strains carrying the GmNMNa promoter-GUS and -GFP binary vectors or the RNAi constructs. Two-week-old soybean shoots cut between the first true leaves and the first trifoliate were placed into rock-wall cubes (Fibrgro, Sarnia, Canada) and inoculated with $4 \mathrm{ml}$ of $A$. rhizogenes carrying the construct of interest (optical density at $600 \mathrm{~nm}\left[\mathrm{OD}_{600}\right]=0.3$ ). After 3 days of drying $\left(23^{\circ} \mathrm{C}, 50 \%\right.$ humidity, long-day conditions), the plants were watered with deionized water. One week later, the plants were transferred to vermiculite/perlite mix (3:1) wetted with nitrogen free-plant nutrient solution (Lullien et al. 1987). After 7 days, the shoots were transferred to the greenhouse $\left(27^{\circ} \mathrm{C}, 20 \%\right.$ humidity, long-day conditions). Finally, the shoots were inoculated with $B$. japonicum $\left(10 \mathrm{ml}, \mathrm{OD}_{600}=0.08\right) 7$ days later. Roots were observed 7 and 28 days after B. japonicum inoculation. Approximately $15 \%$ of the roots were transgenic based on the expression of the reporter genes (i.e., GFP or GUS). The nontransgenic roots were not removed during our experiments to avoid a wounding stress of the composite plants. However, the phenotypes reported were measured only from those roots showing GFP or GUS expression.

$G m N M N a$ promoter-GUS roots were fixed and stained as described by Govindarajulu and associates (2008). Roots with GUS-stained nodules were sectioned and observed as described by Libault and associates (2010c). GmNMNa promoter-GFP roots were observed as described by Libault and associates (2010c).

GmNMNa-RNAi and GUS-RNAi transgenic roots expressing the GFP were isolated 6 weeks after inoculation with $B$. japonicum and nodule numbers were counted using a Leica MZFLIII stereomicroscope equipped with GFP Long Pass filter (500 nm Long Pass, number 41018; Chroma Technology Corp., Rockingham, VT, U.S.A.). SYTO13 staining of GmNMNaRNAi and GUS-RNAi nodule sections was performed as described by Libault and associates (2009).

Transmission electron microscopy of freeze-substituted mature nodules was performed as described by Libault and associates (2010c).

\section{RNA extraction, DNase treatment, reverse transcription, quantitative PCR primer design, quantitative PCR conditions, and data analysis.}

Total RNA isolations, DNase treatments, reverse transcription, and qRT-PCR primer design were performed as described by Libault and associates (2008). The primers used in this study were designed with primer3 software using the criteria described by Libault and associates (2010a). The primer list is available in Supplementary Table 5.

The qRT-PCR tests were performed as described by Libault and associates (2010c) using the geometrical average of Cons4, Cons6, and Cons 7 expression (Libault et al. 2008) to normalize the expression levels of GmNMNa and GmENOD40 genes. Fold changes of gene expression between inoculated and mock-inoculated samples were calculated at the indicated time point during the time course for the three different biological replicates. The average of these fold changes is represented. To statistically validate the differences, a Student's $t$ test between the three inoculated and the three mock-inoculated expression levels was calculated at each time point of the time course.

\section{ACKNOWLEDGMENTS}

This work was funded by a grant from the National Science Foundation (Plant Genome Program, number DBI-0421620). We thank A. Replogle and $\mathrm{S}$. Thibivilliers for critical reading of the manuscript, J. Chang and $\mathrm{Z}$. Wang for their help in the prediction of the RNA-binding domains of GmNMNa protein, Y.-L. Xiao and C. Town for providing the pYXT1 and pYXT2 binary vectors, J. Gruenkemeyer and C. L. Besch Williford (University of Missouri RADIL) for their help on tissue sections, and A. Farmer for his support in using the CMTV software to highlight microsynteny relationship among legumes.

\section{LITERATURE CITED}

Bannai, H., Tamada, Y., Maruyama, O., Nakai, K., and Miyano, S. 2002. Extensive feature detection of N-terminal protein sorting signals. Bioinformatics 18:298-305.

Benedito, V. A., Torres-Jerez, I., Murray, J. D., Andriankaja, A., Allen, S., Kakar, K., Wandrey, M., Verdier, J., Zuber, H., Ott, T., Moreau, S., Niebel, A., Frickey, T., Weiller, G., He, J., Dai, X., Zhao, P. X., Tang, Y., and Udvardi, M. K. 2008. A gene expression atlas of the model legume Medicago truncatula. Plant J. 55:504-513.

Benedito, V. A., Li, H., Dai, X., Wandrey, M., He, J., Kaundal, R., TorresJerez, I., Gomez, S. K., Harrison, M. J., Tang, Y., Zhao, P. X., and Udvardi, M. K. 2010. Genomic inventory and transcriptional analysis of Medicago truncatula transporters. Plant Physiol. 152:1716-1730.

Berg, R. H., and Beachy, R. N. 2008. Fluorescent protein applications in plants. Methods Cell Biol. 85:153-177.

Bonke, M., Thitamadee, S., Mahonen, A. P., Hauser, M. T., and Helariutta, Y. 2003. APL regulates vascular tissue identity in Arabidopsis. Nature 426:181-186.

Cai, C. Z., Han, L. Y., Ji, Z. L., Chen, X., and Chen, Y. Z. 2003. SVMProt: Web-based support vector machine software for functional classification of a protein from its primary sequence. Nucleic Acids Res. 31:3692-3697.

Carrie, C., Kuhn, K., Murcha, M. W., Duncan, O., Small, I. D., O’Toole, N., and Whelan, J. 2009. Approaches to defining dual-targeted proteins in Arabidopsis. Plant J. 57:1128-1139.

Cermola, M., Fedorova, E., Tate, R., Riccio, A., Favre, R., and Patriarca, E. J. 2000. Nodule invasion and symbiosome differentiation during Rhizobium etli-Phaseolus vulgaris symbiosis. Mol. Plant-Microbe Interact. 13:733-741.

Chang, D. D., and Clayton, D. A. 1987. A mammalian mitochondrial RNA processing activity contains nucleus-encoded RNA. Science 235:11781184

Curtis, M. D., and Grossniklaus, U. 2003. A gateway cloning vector set for high-throughput functional analysis of genes in planta. Plant Physiol. 133:462-469

Day, D. A., Price, G. D., and Gresshoff, P. M. 1986. Isolation and oxidative properties of mitochondria and bacteroids from soybean nodules. Protoplasma 134:121-129.

de Castro Silva Filho, M., Chaumont, F., Leterme, S., and Boutry, M. 1996. Mitochondrial and chloroplast targeting sequences in tandem modify protein import specificity in plant organelles. Plant Mol. Biol. 30:769-780.

Ferguson, B. J., and Gresshoff, P. M. 2009. Soybean as a model legume. Grain Legumes Magazine 53:7.

Gold, H. A., Topper, J. N., Clayton, D. A., and Craft, J. 1989. The RNA processing enzyme RNase MRP is identical to the Th RNP and related to RNase P. Science 245:1377-1380.

Govindarajulu, M., Elmore, J. M., Fester, T., and Taylor, C. G. 2008. Evaluation of constitutive viral promoters in transgenic soybean roots and nodules. Mol. Plant-Microbe Interact. 21:1027-1035.

Govindarajulu, M., Kim, S. Y., Libault, M., Berg, R. H., Tanaka, K., Stacey, G., and Taylor, C. G. 2009. GS52 ecto-apyrase plays a critical role during soybean nodulation. Plant Physiol. 149:994-1004.

He, J., Benedito, V. A., Wang, M., Murray, J. D., Zhao, P. X., Tang, Y., and Udvardi, M. K. 2009. The Medicago truncatula gene expression atlas web server. BMC Bioinformatics 10:441.

Høgslund, N., Radutoiu, S., Krusell, L., Voroshilova, V., Hannah, M. A., Goffard, N., Sanchez, D. H., Lippold, F., Ott, T., Sato, S., Tabata, S., Liboriussen, P., Lohmann, G. V., Schauser, L., Weiller, G. F., Udvardi, M. K., and Stougaard, J. 2009. Dissection of symbiosis and organ development by integrated transcriptome analysis of lotus japonicus 
mutant and wild-type plants. PLoS One 4:e6556.

Hwang, S., Gou, Z., and Kuznetsov, I. B. 2007. DP-Bind: A web server for sequence-based prediction of DNA-binding residues in DNA-binding proteins. Bioinformatics 23:634-636.

Indrasumunar, A., Kereszt, A., Miyagi, M., Nguyen, C. D. T., Li, D. X., Searle, I., Men, A., Carroll, B. J., and Gresshoff, P. M. 2010. Inactivation of duplicated Nod-factor receptor 5 (NFR5) genes in recessive loss-of-function non-nodulation mutants of allotetraploid soybean ( $G l y$ cine $\max$ L. Merr.). Plant Cell Physiol. 51:201-214.

Indrasumunar, A., Searle, I., Lin, M. H., Kereszt, A., Men, A., Carroll, B J., and Gresshoff, P. M. 2011. Nodulation factor receptor kinase $1 \alpha$ controls nodule organ number in soybean (Glycine max L. Merr). Plant J. 65:39-50.

Lerouge, P., Roche, P., Faucher, C., Maillet, F., Truchet, G., Promé, J. C., and Dénarié, J. 1990. Symbiotic host specificity of Rhizobium meliloti is determined by a sulphated and acylated glucosamine oligosaccharide signal. Nature 344:781-784

Libault, M., Thibivilliers, S., Bilgin, D. D., Radwan, O., Benitez, M., Clough, S. J., and Stacey, G. 2008. Identification of four soybean reference genes for gene expression normalization. Plant Genet. 1:44-54.

Libault, M., Joshi, T., Takahashi, K., Hurley-Sommer, A., Puricelli, K., Blake, S., Finger, R. E., Taylor, C. G., Xu, D., Nguyen, H. T., and Stacey, G. 2009. Large-scale analysis of putative soybean regulatory gene expression identifies a $M y b$ gene involved in soybean nodule development. Plant Physiol. 151:1207-1220.

Libault, M., Farmer, A., Brechenmacher, L., Drnevich, J., Langley, R. J., Bilgin, D. D., Radwan, O., Neece, D. J., Clough, S. J., May, G. D., and Stacey, G. 2010a. Complete transcriptome of the soybean root hair cell, a single-cell model, and its alteration in response to Bradyrhizobium japonicum infection. Plant Physiol. 152:541-552.

Libault, M., Farmer, A., Joshi, T., Takahashi, K., Langley, R. J., Franklin, L. D., He, J., Xu, D., May, G., and Stacey, G. 2010b. An integrated transcriptome atlas of the crop model Glycine max, and its use in comparative analyses in plants. Plant J. 63:86-99.

Libault, M., Zhang, X. C., Govindarajulu, M., Qiu, J., Ong, Y. T., Brechenmacher, L., Berg, R. H., Hurley-Sommer, A., Taylor, C. G., and Stacey, G. 2010c. A member of the highly conserved FWL (tomato FW2.2-like) gene family is essential for soybean nodule organogenesis. Plant J. 62:852-864.

Lindahl, L., and Zengel, J. M. 1995. RNase MRP and rRNA processing. Mol. Biol. Rep. 22:69-73.

Lodwig, E. M., Leonard, M., Marroqui, S., Wheeler, T. R., Findlay, K., Downie, J. A., and Poole, P. S. 2005. Role of polyhydroxybutyrate and glycogen as carbon storage compounds in pea and bean bacteroids. Mol. Plant-Microbe Interact. 18:67-74.

Lullien, V., Barker, D. G., de Lajudie, P., and Huguet, T. 1987. Plant gene expression in effective and ineffective root nodules of alfalfa (Medicago sativa). Plant Mol. Biol. 9:469-478.

Minami, E., Kouchi, H., Cohn, J. R., Ogawa, T., and Stacey, G. 1996. Expression of the early nodulin, ENOD40, in soybean roots in response to various lipo-chitin signal molecules. Plant J. 10:23-32.

Mitschke, J., Fuss, J., Blum, T., Hoglund, A., Reski, R., Kohlbacher, and Rensing, S. A. 2009. Prediction of dual protein targeting to plant organelles. New Phytol. 183:224-235.

Nelson, B. K., Cai, X., and Nebenfuhr, A. 2007. A multicolored set of in vivo organelle markers for co-localization studies in Arabidopsis and other plants. Plant J. 51:1126-1136.

Newcomb, E. H., Tandon, D. R., and Kowal, R. R. 1985. Ultrastructural specialization for ureide production in uninfected cells of soybean root nodules. Protoplasma 125:1-12

Oldroyd, G. E., and Downie, J. A. 2008. Coordinating nodule morphogenesis with rhizobial infection in legumes. Annu. Rev. Plant Biol. 59:519-546.

Ronson, C. W., Lyttleton, P., and Robertson, J. G. 1981. C(4)-dicarboxylate transport mutants of Rhizobium trifolii form ineffective nodules on Trifolium repens. Proc. Natl. Acad. Sci. U.S.A. 78:4284-4288.

Salamov, A., and Solovyev, V. 2000. Ab initio gene finding in Drosophila genomic DNA. Genome Res. 10:516-522.

Sawkins, M. C., Farmer, A. D., Hoisington, D., Sullivan, J., Tolopko, A., Jiang, Z., and Ribaut, J. M. 2004. Comparative map and trait viewer
(CMTV): An integrated bioinformatic tool to construct consensus maps and compare QTL and functional genomics data across genomes and experiments. Plant Mol. Biol. 56:465-480.

Schmutz, J., Cannon, S. B., Schlueter, J., Ma, J., Mitros, T., Nelson, W. Hyten, D. L., Song, Q., Thelen, J. J., Cheng, J., Xu, D., Hellsten, U., May, G. D., Yu, Y., Sakurai, T., Umezawa, T., Bhattacharyya, M. K., Sandhu, D., Valliyodan, B., Lindquist, E., Peto, M., Grant, D., Shu, S., Goodstein, D., Barry, K., Futrell-Griggs, M., Abernathy, B., Du, J., Tian, Z., Zhu, L., Gill, N., Joshi, T., Libault, M., Sethuraman, A., Zhang, X. C., Shinozaki, K., Nguyen, H. T., Wing, R. A., Cregan, P., Specht, J., Grimwood, J., Rokhsar, D., Stacey, G., Shoemaker, R. C. and Jackson, S. A. 2010. Genome sequence of the palaeopolyploid soybean. Nature 463:178-183.

Schwacke, R., Fischer, K., Ketelsen, B., Krupinska, K., and Krause, K. 2007. Comparative survey of plastid and mitochondrial targeting properties of transcription factors in Arabidopsis and rice. Mol. Genet. Genomics 277:631-646.

Severin, A. J., Woody, J. L., Bolon, Y. T., Joseph, B., Diers, B. W., Farmer A. D., Muehlbauer, G. J., G. J. Nelson, G. J., Grant, D., Specht, J. E., Graham, M. A., Cannon, S. B., May, G. D., Vance, C. P., and Shoemaker, R. C. 2010. RNA-Seq Atlas of Glycine max: A guide to the soybean transcriptome. BMC Plant Biol. 10:160.

Tollervey, D. 1995. Genetic and biochemical analyses of yeast RNase MRP. Mol. Biol. Rep. 22:75-79.

Trainer, M. A., and Charles, T. C. 2006. The role of PHB metabolism in the symbiosis of rhizobia with legumes. Appl. Microbiol. Biotechnol. 71:377-386.

Udvardi, M. K., and Day, D. A. 1997. Metabolite transport across symbiotic membranes of legume nodules. Annu. Rev. Plant Physiol. Plant Mol. Biol. 48:493-523.

Veereshlingam, H., Haynes, J. G., Penmetsa, R. V., Cook, D. R., Sherrier, D. J., and Dickstein, R. 2004. nip, a symbiotic Medicago truncatula mutant that forms root nodules with aberrant infection threads and plant defense-like response. Plant Physiol. 136:3692-3702.

Wang, L., and Brown, S. J. 2006. BindN: A web-based tool for efficient prediction of DNA and RNA binding sites in amino acid sequences. Nucleic Acids Res. 34:W243-248.

Wang, C., Saldanha, M., Sheng, X., Shelswell, K. J., Walsh, K. T., Sobral, B. W., and Charles, T. C. 2007. Roles of poly-3-hydroxybutyrate (PHB) and glycogen in symbiosis of Sinorhizobium meliloti with Medicago sp. Microbiology 153:388-398.

Wong, P. P., and Evans, H. J. 1971. Poly-beta-hydroxybutyrate utilization by soybean (Glycine max Merr.) nodules and assessment of its role in maintenance of nitrogenase activity. Plant Physiol. 47:750-755.

Xiao, Y. L., Smith, S. R., Ishmael, N., Redman, J. C., Kumar, N., Monaghan, E. L., Ayele, M., Haas, B. J., Wu, H. C., and Town, C. D. 2005. Analysis of the cDNAs of hypothetical genes on Arabidopsis chromosome 2 reveals numerous transcript variants. Plant Physiol. 139:1323-1337.

Yan, C., Terribilini, M., Wu, F., Jernigan, R. L., Dobbs, D., and Honavar, V. 2006. Predicting DNA-binding sites of proteins from amino acid sequence. BMC Bioinformatics 7:262.

\section{AUTHOR-RECOMMENDED INTERNET RESOURCES}

The Arabidopsis Information Resource database: http://www.arabidopsis.org/Blast/index.jsp

iPSORT web server: ipsort.hgc.jp

The Joint Genome Institute Soybean (Glycine max) website: www.phytozome.net/soybean.php

Kazusa DNA Research Lotus japonicus webpage www.kazusa.or.jp/lotus

Medicago genome assembly website: www.medicagohapmap.org/?genome

Primer3 software: biotools.umassmed.edu/bioapps/primer3 www.cgi

PSORT prediction server: psort.hgc.jp/form.html

Softberry MaliP software: linux1.softberry.com/berry.phtml

SVM-Prot Protein Functional Family Prediction server: jing.cz3.nus.edu.sg/cgi-bin/svmprot.cgi 